EPJ Web of Conferences 41, 10017 (2013)

DOI: $10.1051 /$ epjconf/20134110017

(C) Owned by the authors, published by EDP Sciences, 2013

\title{
All-fiber femtosecond Cherenkov source
}

\author{
X. Liu ${ }^{1}$, J. Lægsgaard ${ }^{1}$, U. Møller ${ }^{1}$, H. Tu ${ }^{2}$, S. A. Boppart ${ }^{2}$, and D. Turchinovich ${ }^{1,3, a}$ \\ 1 DTU Fotonik, Technical University of Denmark, DK-2800 Kgs. Lyngby, Denmark \\ 2 Biophotonics Imaging Laboratory, University of Illinois at Urbana-Champaign, Urbana, Illinois \\ 61801, U.S.A. \\ 3 Max Planck Institute for Polymer Research, Ackermannweg 10, 55128 Mainz, Germany
}

\begin{abstract}
An all-fiber femtosecond Cherenkov radiation source is demonstrated for the first time, to the best of our knowledge. Using a stable monolithic femtosecond $\mathrm{Yb}$ doped fiber laser as the pump source, and the combination of photonic crystal fibers as the wave-conversion medium, we have generated tunable Cherenkov radiation at visible wavelengths 580 - $630 \mathrm{~nm}$, with pulse duration of sub- $160 \mathrm{fs}$, and the $3 \mathrm{~dB}$ spectral bandwidth not exceeding $36 \mathrm{~nm}$. Such femtosecond source can find applications in practical biophotonics such as bio-imaging and microscopy.
\end{abstract}

Many applications in biophotonics, such as multiphoton microscopy and ultrafast spectroscopy, require narrowband ultrafast $(<5 \mathrm{ps})$ pulses of a few milliwatts of average power that are tunable across the NIR, VIS, and UV spectral ranges $[1,2]$. In-fiber Cherenkov radiation (CR), also known as dispersive wave generation or nonsolitonic radiation, and originating from the perturbation of a stable temporal soliton by the higher-order fiber dispersion, can provide such a tunable output at multimilliwatt signal levels [3]. However, to the best of our knowledge, all the reported systems for femtosecond

a e-mail: dmtu@fotonik.dtu.dk

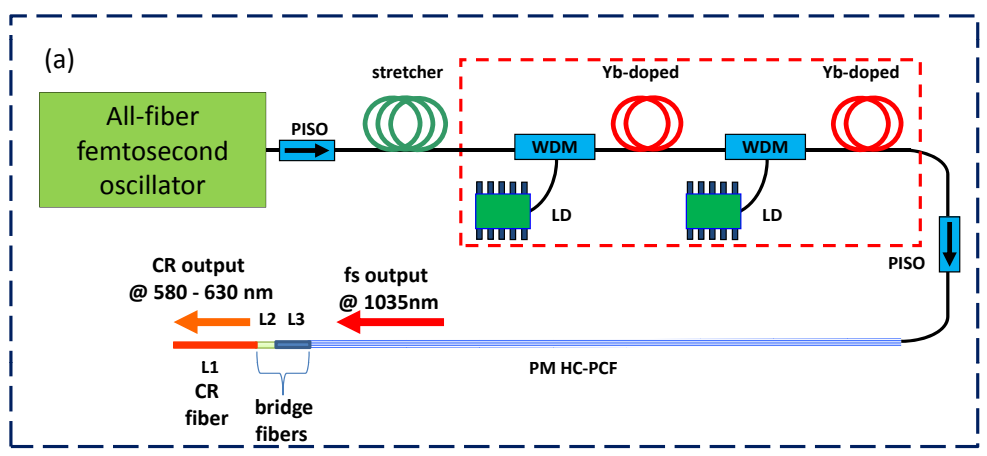

(b)

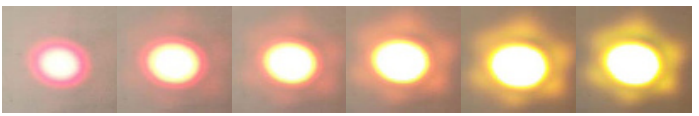

Fig. 1. (a) Layout of the all-fiber Cherenkov source: PISO - polarization-maintaining isolator, WDM - 980/1030 wavelength division multiplexer, LD - single-mode pumping diode at $974 \mathrm{~nm}$, HC-PCF - laser pulse compressor hollow-core photonic crystal fiber, CR fiber - highly nonlinear fiber for CR generation. (b) From left to right: the far-field saturated visible images of the generated Cherenkov output as the pump laser power increases from 150 $\mathrm{mW}$ to $300 \mathrm{~mW}$.

This is an Open Access article distributed under the terms of the Creative Commons Attribution License 2.0, which permits unrestricted use, distribution, and reproduction in any medium, provided the original work is properly cited. 

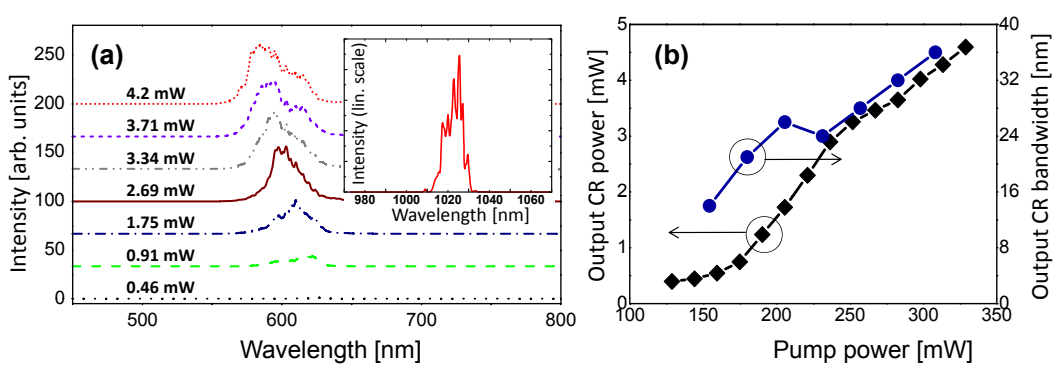

Fig. 2. (a) The spectra of $C R$ at variable output power. Inset: The spectrum of the input femtosecond pump signal. (b) The generated CR output power (black diamonds) and $3 \mathrm{~dB}$ bandwidth (blue circles) as a function of increasing pump power.

fiber-generated CR were based on free-space coupling, and used the solid-state lasers as pumps, naturally causing environmental instability. They are thus not suitable for the real-life biophotonics applications outside of the lab. In this work, we demonstrate an all-fiber laser system generating milliwatt average power, sub-160 fs, and spectrally isolated CR pulses at visible wavelength. Such a monolithic, all-fiber CR system provides a very high environmental and operational stability, defined by that of a self-stabilized Yb-doped fiber mode-locked laser used as a pump.

The complete ultrafast Cherenkov system is shown in Fig 1.(a). It consists of two parts: an all-fiber $\mathrm{nJ}$-level highly-stable $\mathrm{Yb}$-fiber laser operating at $26.7 \mathrm{MHz}$ repetition rate and delivering the $1035 \mathrm{~nm}$ femtosecond pulses as the pump source; and a spliced-on nonlinear fiber link for Cherenkov wave generation. The details of the pump laser can be found in $[4,5]$ and references therein. The small-core nonlinear fiber NL-3.0-850, which has the zero dispersion wavelength (ZDW) around $850 \mathrm{~nm}$, is used for CR generation. In order to minimize the transmission loss between the NL-3.0-850 fiber and the hollow-core anomalous dispersion photonic crystal fiber (HC-PCF) delivering the pulse-compressed pump laser signal, two other types of fibers are spliced in between them as bridge fibers. The modefield diameters (MFD) of the NL-3.0-850 fiber, the two bridge fibers, and the HC-PCF are $1.8 \mu \mathrm{m}$, $3.3 \mu \mathrm{m}, 6.6 \mu \mathrm{m}$ and $7.5 \mu \mathrm{m}$ respectively, and the total splice loss in the CR link is around $4 \mathrm{~dB}$. The NL-3.0-850 fiber and the two bridge fibers are $10 \mathrm{~cm}$ (L1), $2 \mathrm{~mm}$ (L2) and $8 \mathrm{~cm}$ (L3) long, respectively.

The pump pulses used in our experiment had an autocorrelation full width at half maximum (FWHM) of $460 \mathrm{fs}$ when fully compressed [4]. In our system they were negatively pre-chirped, in order to provide the shortest pulse duration while in the NL-3.0-850 fiber, since the dispersion of the bridge fibers used in the link is normal. In this way the strongest CR output can be generated. The far-field saturated visible images of the CR emitted from our system are shown in Fig. 1(b), exhibiting the typical profile of the 7-cell PCF mode.

During the propagation in the small-core PCF, the pump pulses initially undergo nonlinear pulse compression due to the combination of self-phase-modulation (SPM) and anomalous dispersion. Around the point of maximal compression, the ultrafast visible CR pulses are generated. Fig. 2(a) shows the emitted CR output spectra measured at different output powers and the inset shows the typical spectrum of the input pump pulse.

The generated CR wavelength is dependent on the peak power of the input pump pulse due to the CR phase-matching condition. In our experiments, when the $1035 \mathrm{~nm}$ pump power was increasing, the central wavelength of the emitted CR can shift from $630 \mathrm{~nm}$ to $580 \mathrm{~nm}$, yielding the blue-shift as expected [6]. The $3 \mathrm{~dB}$ bandwidth of the emitted CR as a function of the input pump power is shown as the blue circular line in Fig. 2(b). When the input $1035 \mathrm{~nm}$ pump power increases from $154 \mathrm{~mW}$ to $308 \mathrm{~mW}$, the $3 \mathrm{~dB}$ bandwidth of CR increases from $14 \mathrm{~nm}$ to $36 \mathrm{~nm}$. The increase in CR bandwidth with increasing pump power can be ascribed to both SPM/XPM effects, and Raman-induced red-shift of the pump pulses. The wavelength tunability is realized by the control of the chirp and bandwidth of $1035 \mathrm{~nm}$ pump pulse with controlling the inversion in the amplifier section of the Yb-fiber laser (marked by a red dashed box in Fig. 1 (a)) [7]. 


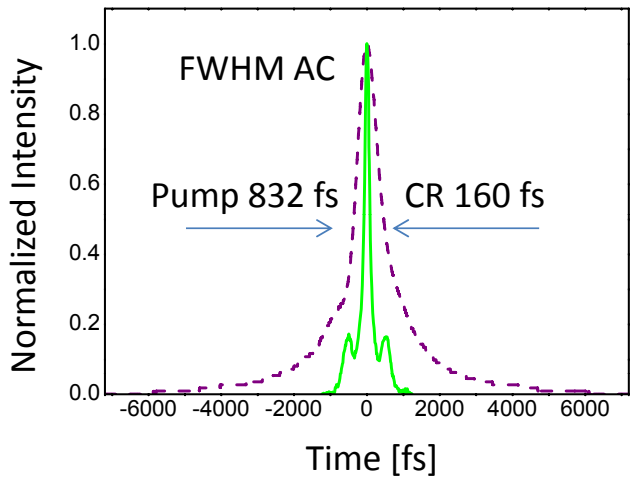

Fig. 3. Autocorrelation of the generated CR pulse (green solid line) with the output power of $1.7 \mathrm{~mW}$, and of the input pump pulse (purple dashed line).

The black diamond line in Fig. 2(b) shows the emitted CR power as a function of the average input pump power. When the input pump power is $130 \mathrm{~mW}$, the CR output shows a generation threshold. When the pump power reaches $180 \mathrm{~mW}$, the CR conversion efficiency grows significantly, and after that the Cherenkov conversion efficiency saturates. We note here that the generated CR power is also dependent on the polarization state of the NL-3.0-850 [2], and highest CR output is generated by optimal rotational orientation of the PCF during the fiber splicing process.

The temporal profile of the generated CR pulses was characterized by autocorrelation (AC). The $\mathrm{AC}$ of the $\mathrm{CR}$ with the output power of $1.7 \mathrm{~mW}$ and the $\mathrm{AC}$ of the input pump pulses are shown in Fig. 3 , as the solid and dashed line respectively. The FWHM of AC trace of the input $1035 \mathrm{~nm}$ pump pulse is $832 \mathrm{fs}$, whereas the FWHM of the AC trace for the generated CR pulse is 160 fs, i.e. more than 5 times shorter, as a consequence of nonlinear pump pulse compression in the CR fiber link. The actual duration of the CR pulse is even shorter than its AC FWHM value. When the CR signals emitted in the output power range from $1 \mathrm{~mW}$ to $2.5 \mathrm{~mW}$, the AC FWHM are in the range $145-225 \mathrm{fs}$. From the simulations $[8,9]$ we can also estimate the pulse duration of the CR output generated from $10 \mathrm{~cm}$ NL-3.0-850 to be on the order of hundreds of femtoseconds, which matches our experimental results.

As a conclusion, in this work we have shown an all-fiber femtosecond Cherenkov radiation source with convenient electrical tunability in the range $580 \mathrm{~nm}-630 \mathrm{~nm}$, and multi-milliwatt output powers (see [10] for more details). This laser source is highly stable: as an example of stable operation, the CR output shown in Fig. 3 remains unchanged during more than one month-long operation of our system, during which over 200 on-off cycles for the whole system occurred. We believe that such a stability of our system makes it promising for practical biophotonics applications, e.g. ultrafast spectroscopy, fluorescence lifetime imaging, and multiphoton microscopy etc, performed in the out-of-the-lab environments.

\section{References}

1. J. C. Knight and D. V. Skryabin, Opt. Express 15, 15365 (2007)

2. H. Tu and S. A. Boppart, Opt. Express 17, 9858 (2009)

3. N. Akhmediev and M. Karlsson, Phy. Rev. A 51, 2602 (1995)

4. X. Liu, J. Lægsgaard, and D. Turchinovich, Opt. Express 18, 15475 (2010)

5. X. Liu, J. Lægsgaard, and D. Turchinovich, J. Sel. Top. Quant. Electron., in press

6. G. Chang, L. J. Chen, and F. X. Kärtner, Opt. Lett. 35, 2361 (2010)

7. D. Turchinovich, X. Liu, and J. Lægsgaard, Opt. Express 16, 14004 (2008)

8. I. Cristiani, R. Tediosi, L. Tartara, and V. Degiorgio, Opt. Express 12, 124 (2004)

9. D. R. Austin, C. M. de Sterke, B. J. Eggleton, and T. G. Brown, Opt. Express 14, 11997 (2006)

10. X. Liu, J. Lægsgaard, U. Møller, H. Tu, S. A. Boppart, and D. Turchinovich, Opt. Lett. 37, 2769

(2012) 